\title{
EFICÁCIA DE NÚCLEO HOMEOPÁTICO NA PREVENÇÃO DA INFESTAÇÃO POR Dermatobia hominis E Haematobia irritans EM BOVINOS
}

\section{(Evaluation of homeopathic nuclei efficacy on the prevention of infestation by Dermatobia hominis and Haematobia irritans in bovines)}

\author{
PINTO, S.B. ${ }^{1}$; VALENTIM-ZABOTT, M. ${ }^{1}$; ROCHADELLI, R. ${ }^{1}$; VENDRUSCOLO, E.C.G. ${ }^{1}$; FERNANDES, \\ N.L. ${ }^{2}$; FREITAG A.C. ${ }^{3}$; MONTANUCCI, C. ${ }^{3}$; LESSKIU. P.E. ${ }^{4}$, SPESSATTO, D.D. ${ }^{5}$
}

\begin{abstract}
${ }^{1}$ Docentes da UFPR - Campus Palotina; ${ }^{2}$ Doutorando do Curso de Pós Graduação em Processos Biotecnológicos da UFPR, área de concentração Saúde Animal e Humana;

${ }^{3}$ Servidores Técnicos da UFPR - Campus Palotina; ${ }^{4}$ Medico Veterinário; ${ }^{5}$ Mestranda do Curso de Pós Graduação em Ciências Veterinárias da UFPR, área de concentração Patologia Veterinária.
\end{abstract}

RESUMO - O presente trabalho foi desenvolvido na UFPR - Campus Palotina - Paraná e em uma propriedade rural do município de Mundo Novo - Mato Grosso do Sul, durante o período de maio de 2002 a abril de 2003. O experimento foi delineado com os objetivos de avaliar: a) a eficácia do núcleo homeopático (nosódicos $\mathrm{CH} 12$, sacarose e Bixa orelhana) na prevenção da infestação natural por larvas de $D$. hominis e $H$. irritans em bovinos de corte e b) a dinâmica populacional de $D$. homins e $H$. irritans ao longo do ano. Para sua realização foram utilizados 24 vacas, de aproximadamente 15 meses de idade, distribuídas em dois grupos de 12 animais, com pesos médios semelhantes. Os animais do grupo " $A$ " foram tratados com núcleo homeopático adicionado à suplementação mineral na proporção de $400 \mathrm{~g}$ de núcleo adicionado em $25 \mathrm{Kg}$ da mesma. Os animais do grupo "B" receberam suplementação mineral (sem adição de núcleo homeopático). Foram contadas um total de 451 larvas de $D$. hominis. A análise de variância demonstrou diferença estatística significativa $(p<0,01)$ para os valores de contagem total de nódulos de "berne" entre os animais do grupo "A" e "B". Os dados foram submetidos ao teste do $\chi 2$ (qui-quadrado), onde constatou-se que os animais do grupo "A" apresentaram os menores valores de contagem total de nódulos. Os resultados obtidos sugerem que, nas condições do experimento, o núcleo homeopático apresentou efeito preventivo sobre o parasitismo por larvas de $D$. hominis. Foram contados um total de $41.968,00$ adultos de $H$. irritans. Analisadas as freqüências ficou demonstrado que houve diferença estatística $(p<0,01)$ quando analisados pelo teste do $\chi 2$. A intensidade de $H$. irritans foi maior nos animais do grupo "A". Os resultados obtidos sugerem que, nas condições do experimento, o núcleo homeopático não apresentou efeito preventivo sobre o parasitismo por $H$. irritans. Os índices mensais de infestação por $D$. hominis e $H$. irritans entre os grupos tratado e não tratado foram semelhantes durante todo o período experimental, não havendo diferença estatística $(p<0,05)$. Nos meses de outubro e novembro verificou-se os maiores índices de infestação por larvas de $D$. hominis. Na primavera e no verão ocorrerem os maiores índices de infestação por $H$. irritans e os menores no outono e no inverno.

Palavras-chave: Dermatobia hominis, Haematobia irritans, bovinos, homeopatia, prevenção.

ABSTRACT - This study was experimentally conducted at the UFPR-Campus Palotina and in a commercial farm from Mundo Novo county, MS from May, 2002 to April, 2003. The aim was to evaluate the efficacy of a homeopathic nucleus (nosodices $\mathrm{CH} 12$, sucroses and Bixa orelhana) in the prevention of natural infestation by $D$. hominis larvae and $H$. irritans in bovines as well as the 
monthly fluctuation of $D$. hominis and $H$. irritans. In this study, 24 cows of approximately 15 years old and similar weight were distributed in two groups of 12 animals each. Group A was treated with 400 $\mathrm{g}$ of homeopathic nucleus added to $25-30 \mathrm{Kg}$ of mineral salt supplementation. Group B was the control, with no homeopathic nucleus added. A total of $451 \mathrm{D}$. hominis larvae were counted. Group A showed statistically lower values $(p<0.01)$ in the total nodule counting. The results suggest that homeopathic nucleus have a preventive effect over the ectoparasitism of $D$. hominis. A total of 41,968 flies were counted. The frequencies of $H$. irritans were analyzed and showed a statistically higher infestation intensity $(p<0.01)$ in animals from group $B$. The results suggest that homeopathic nucleus does not have a preventive effect over the ectoparasitism of $H$. irritans. The monthly rates of $D$. hominis and $H$. irritans infestation of groups $A$ and $B$ were similar and no statistical difference was observed. The higher infestation by $D$. hominis larval was observed during October and November. The highest period for $H$. irritans infestation was on summer and spring.

Key-words: Dermatobia hominis, Haematobia irritans, bovines, homeopathic, prevention.

\section{Introdução}

Dentre os ectoparasitos de importância médico-veterinária para a América Latina estão a Dermatobia hominis (LINNAEUS JR., 1781) (Diptera: Oestridae) e a Haematobia irritans (Diptera: Muscidae), conhecidas popularmente por "berne" e "mosca-dos-chifres", respectivamente. Os prejuízos econômicos gerados pelos referidos parasitos em bovinos são traduzidos pela diminuição da produção de leite e de carne, pelo baixo ganho de peso e pela dificuldade de aclimação de raças selecionadas. O couro é o subproduto que maior depreciação sofre, levando a sua desvalorização comercial ou inadequação à industrialização (OLIVEIRA, 1991; PINTO et al. 2002a; PINTO et al.; 2002b).

O controle destas parasitoses atualmente é feito apenas com produtos químicos, entretanto, este tipo de controle pode deixar resíduos no ambiente e no animal (GOMES et al. 1988; PINTO et al. 2002b). A demanda crescente por alimentos de origem orgânica exige dos produtores a busca de alternativas de controle de parasitos que não agridam a qualidade da carne e o ambiente. Neste contexto, a homeopatia visa buscar meios de controle dos principais parasitos internos e externos dos bovinos, que sejam eficientes e menos agressivos aos animais e ao ambiente. Controlar as enfermidades e parasitoses que causam grandes prejuízos na bovinocultura com menor custo, possuir um rebanho mais resistente e trabalhar com menor risco de contaminação ambiental, são importantes metas dos bovinocultores. Segundo ESCOSTEGUY (2003), os custos dos tratamentos homeopáticos são cinco vezes menores que os custos dos tratamentos convencionais.

O fato de haver poucas informações, apoiadas na pesquisa, de que o núcleo homeopático adicionado ao sal mineral previne, efetivamente, as infestações por $D$. hominis e $H$. irritans, justificou a execução do presente trabalho. Assim, com vistas ao conhecimento da prevenção da infestação por $D$. hominis e $H$. irritans em bovinos na região Sul do estado do Mato Grosso do Sul foi realizado um experimento com os objetivos de avaliar: a) a eficácia do núcleo homeopático na prevenção da infestação natural por $D$. hominis e $H$. irritans em bovinos nelorados (alto grau de sangue nelore) e b) a dinâmica populacional de $D$. hominis e $H$. irritans em bovinos, ao longo do ano.

\section{Material e Método}

A parte experimental do presente trabalho foi desenvolvida numa propriedade de bovinos de corte situada no município de Mundo Novo, Mato Grosso do Sul, Brasil, no período compreendido entre maio de 2002 a abril de 2003.

Para sua realização foram utilizadas 24 vacas da raça Nelore, de aproximadamente 15 meses e peso médio inicial de $179 \mathrm{Kg}$, criadas em regime extensivo, devidamente 
A eficácia do núcleo homeopático na prevenção da infestação por Dermatobia hominis...

identificadas com brincos. No dia do cadastramento dos animais, realizou-se uma pesagem, sendo que os mesmos foram distribuídos em dois grupos de 12 animais, com pesos médios semelhantes. Em seguida sorteou-se os grupos de acordo com os seguintes tratamentos: a) Grupo "A" tratamento e b) Grupo "B" - testemunha.

Os animais do grupo " $A$ " foram tratados com núcleo homeopático (nosódios $\mathrm{CH} 12$, sacarose e Bixa orelhana) adicionado à suplementação mineral na proporção de $400 \mathrm{~g}$ de núcleo adicionado em $25 \mathrm{Kg}$ da mesma. Os animais do grupo "B" receberam suplementação mineral sem adição de núcleo homeopático. A composição mineral foi fornecida ad libitum aos animais, em cochos cobertos, durante todo o período experimental. $\mathrm{Na}$ época da formação dos grupos, os animais não apresentavam ectoparasitos e durante a fase experimental não sofreram tratamento antiparasitário. Na ocasião do experimento, os animais dos respectivos lotes foram mantidos em pastagem natural, formada de Brachiaria brizantha e Brachiaria decumbens, comum a todos.

Para o estudo da prevenção da infestação por $D$. hominis, mensalmente os animais eram levados ao curral, em intervalos de aproximadamente 28 dias, durante um período de 12 meses, onde foram pesados, examinados pelos recursos semiológicos da palpação e inspeção, e a população de "berne" contada e mapeada conforme sua distribuição no corpo do hospedeiro. Para o estudo da prevenção da infestação por $H$. irritans, mensalmente, em intervalos de aproximadamente 14 dias, durante um período de 12 meses, os animais foram levados (durante o período da manhã) ao curral, onde, pela inspeção, a população de "mosca-dos-chifres" foi contada. A contagem de $H$. irritans na superfície corpórea dos bovinos foi realizada apenas na lateral direita do animal e o valor obtido foi multiplicado por dois.

Para o estudo da dinâmica populacional de $D$. hominis e $H$. irritans foi considerada a média do total de "bernes" e de "mosca-dos-chifres" encontrados mensalmente em bovinos ao longo do ano. Ainda durante o período experimental, foram colhidas amostras de sangue e amostras de fezes. Basicamente, os exames hematológicos e coproparasitológicos foram executados para acompanhar o estado sanitário dos animais, pois a produção pecuária depende diretamente do estado de saúde dos mesmos. Como procedimento de rotina, a maioria dos bovinocultores brasileiros faz uso de fármacos antiparasitários, entretanto, durante a realização do presente trabalho os animais do grupo "B" não receberam nenhum tipo de fármaco antiparasitário. Desta forma, os exames hematológicos e coproparasitológicos foram de suma importância para o controle do estado sanitário, pois animais altamente parasitados podem apresentar sinais característicos da Hipersensibilidade do Tipo I, incluindo eosinofilia, edema, asma e dermatite, bem como a produtividade pode ser reduzida, tanto temporariamente como permanentemente. Segundo REY (2001), a eosinofilia é particularmente acentuada nas doenças alérgicas, nas infecções parasitárias principalmente nas helmintíases, e nas reações a determinados fármacos. De acordo com HOFFMANN (1987), a quantidade de ovos de parasitos nas fezes depende de numerosos fatores, como por exemplo: a) período do dia em que é feita a coleta de fezes; b) idade, nutrição, manejo, imunidade e alterações fisiológicas e patológicas do trato digestório do hospedeiro; c) reinfecções do hospedeiro; d) número de parasitos do hospedeiro; e) relação de parasitos machos e fêmeas; f) diferentes quantidades de ovos postos por diversos tipos de parasitos; g) idade do parasito e h) patogenicidade do parasito.

Os dados meteorológicos de temperatura e precipitação pluvial foram obtidos junto ao Instituto Tecnológico SIMEPAR.

Para análise da eficácia do medicamento homeopático foi aplicada a análise de variância e, o teste de Tukey ao nível de $p<0,05$. O teste do $\chi 2$, segundo uma tabela de contigência, foi utilizado para a análise do grau de significância $(p<0,05)$ entre a freqüência de larvas de "berne" e de "mosca-dos-chifres" nos diferentes grupos (VIEIRA, 1983).

\section{Resultados e Discussão}

A análise de variância demonstrou diferença estatística significativa $(p<0,01)$ para os valores de contagem total de nódulos de larvas de $D$. 
hominis entre os animais do grupo "A" e " $\mathrm{B}$ ". Os dados foram submetidos ao teste do $\chi 2$, onde constatou-se que os animais do grupo " $A$ " apresentaram os menores valores de contagem total de nódulos (TABELA 1). Os resultados verificados na contagem de nódulos de larvas de $D$. hominis nos grupos tratado e não tratado pelo núcleo homeopático, indicaram que nas condições do experimento, o núcleo homeopático apresentou ação preventiva sobre o parasitismo do "berne", e que, portanto, a adição de núcleo ao sal mineral é uma prática eficiente para o controle desse ectoparasito. GOMES et al. (1988), ao estudarem a distribuição corporal e a sazonalidade das larvas de $D$. hominis em bovinos nelorados, no município de Rio Brilhante, MS, tratados ou não com flor de enxofre, verificaram que o enxofre não forneceu efeito bernecida quando fornecido juntamente com a mistura mineral.

TABELA 1 - TESTE DO $\chi 2$ PARA INCIDÊNCIA DE LARVAS DE Dermatobia hominis EM BOVINOS TRATADOS COM NÚCLEO HOMEOPÁTICO E CONTROLES, NO PERÍODO DE MAIO DE 2002 A ABRIL DE 2003.

\begin{tabular}{lccc}
\hline Animais & Freqüência Observada & Freqüência Esperada & $\chi 2$ \\
\hline Tratados & $145(0,32)$ & $225,5(0,50)$ & 14,37 \\
Controles & $306(0,68)$ & $225,5(0,50)$ & 14,37 \\
\hline Total & $451(1,00)$ & $451(1,00)$ & 28,74 \\
\hline
\end{tabular}

Teste do Qui-quadrado, ao nível de significância de $p<0,01^{\text {** }}$

A análise de variância demonstrou diferença estatística significativa $(p<0,01)$ para os valores de contagem total de "mosca-dos-chifres" entre os animais do grupo "A" e "B". Os dados foram submetidos ao teste do c2 (qui-quadrado), onde constatou-se que os animais do grupo " $A$ " apresentaram os maiores valores de contagem total de moscas (TABELA 2), revelando ser, nas condições do experimento, uma prática não eficiente na prevenção da infestação por $H$. irritans em bovinos. Melhores resultados, provavelmente, poderiam ser obtidos usando-se em conjunto o núcleo homeopático adicionado ao sal mineral, a introdução de Onthophagus gazella na propriedade e ao uso de armadilhas para captura de "mosca-dos-chifres". SUTHERST e TOZER (1995) e TOZER e SUTHERST (1996), verificaram, na Florida eAustrália, bons resultados no controle de $H$. irritans utilizando armadilhas, constituídas de uma tenda de plástico, instalada na entrada de currais ou em outros pontos de passagem obrigatória.

TABELA 2 - TESTE DO c2 PARA INCIDÊNCIA DE Haematobia irritans EM BOVINOS TRATADOS COM NÚCLEO HOMEOPÁTICO E CONTROLES, NO PERÍODO DE MAIO DE 2002 A ABRIL DE 2003.

\begin{tabular}{lccc}
\hline Animais & Freqüência Observada & Freqüência Esperada & $\chi 2$ \\
\hline Tratados & $22.232,00(0,53)$ & $20.984,00(0,50)$ & 77,23 \\
Controles & $19.736,00(0,47)$ & $20.989,00(0,50)$ & 77,23 \\
\hline Total & $41.968,00(1,00)$ & $41.968,00(1,00)$ & 144,45 \\
\hline
\end{tabular}

Teste do Qui-quadrado, ao nível de significância de $p<0,01^{\text {** }}$ 
A eficácia do núcleo homeopático na prevenção da infestação por Dermatobia hominis...

O número de leucócitos totais dos animais do grupo "A" oscilou entre $6.950 / \mu \mathrm{l}$ e $34.300 \mu \mathrm{l}$, nos animais do grupo "B" oscilou entre 6.600/ $\mu \mathrm{l}$ e $27.100 / \mu \mathrm{l}$; o que supera os valores de referência para a espécie, 4.000 e $12.000 / \mu l$ (JAIN, 1993). Segundo DITTRICH et al. (1996), os resultados laboratoriais podem sofrer alterações devido a condições de criação dos animais, tipo de alimentação, idade, sexo, raça e condições patológicas subclínicas.

Nos exames coproparasitológicos dos animais (grupos " $A$ " e "B") foram observados ovos de tipo estrongiliformes, da classe Nematoda; ovos da Classe cestoda e oocistos da classe Coccidia. Em relação aos ovos da Superfamília Strongyloidea, pode-se constatar que os mesmos estiveram presentes durante os 12 meses do período experimental. Ao analisar todos os exames realizados, observouse que o número de ovos de estrogilídioeos por grama de fezes oscilou de 0 a 500 opg (ovos de helmintos por grama de fezes). O teste de Tukey mostrou que não ocorreu diferença significativa $(p<0,05)$ no opg dos animais ao longo do período experimental.

As oscilações climáticas verificadas no decorrer do trabalho encontram-se dentro dos limites favoráveis ao desenvolvimento das fases de vida livre de $D$. hominis e $H$. irritans. Quanto à sazonalidade de larvas de $D$. hominis em bovinos houve infestações ao longo de todo o período experimental, tendo sido contados 451 nódulos de "berne". Os picos populacionais de larvas de $D$. hominis em bovinos, ocorreram quando a temperatura média, em ascensão, atingia $30^{\circ} \mathrm{C}$, e a população decresceu com temperatura inferior a $23^{\circ} \mathrm{C}$. O pico máximo de infestação por larvas foi observado nos meses de outubro e novembro. Nestes meses foi constatado um total de 266 nódulos, o que representa uma freqüência relativa de $59 \%$ do total de larvas contadas no período analisado. Comportamento semelhante foi observado por GOMES et al. (1988) no município de Rio Brilhante, MS. Segundo os autores os maiores picos populacionais ocorreram no período compreendido entre os meses de agosto a outubro e durante o mês de dezembro. GOMES et al. (1996), ao avaliarem a intensidade parasitária de larvas de $D$. hominis em bovinos de diferentes raças criadas extensivamente na região de cerrado em Mato Grosso do Sul, verificaram maiores picos de parasitismo nos meses de março a maio, período chuvoso, seguido de picos menores em agosto e setembro, fim do período seco e início do chuvoso. GOMES (2001), ao estudar as espécies de Fanniidae vetoras de ovos de $D$. hominis na região de Campo Grande, MS, observou um aumento de infestação desse parasito em bovinos nos meses de setembro a outubro. PINTO et al. (2002a), ao estudar a dinâmica populacional de larvas de $D$. hominis em bovinos de leite no município de Palotina, Paraná, observaram presença significativa de larvas em setembro e outubro.

Quanto à dinâmica populacional de $H$. irritans em bovinos houve infestações ao longo de todo o período experimental, tendo sido contados 41.968,00 exemplares. Os picos populacionais de $H$. irritans em bovinos, ocorreram quando a temperatura média, em ascensão, atingia $24^{\circ} \mathrm{C}$, e a população decresceu com temperatura média inferior a $20^{\circ} \mathrm{C}$. O pico máximo de infestação foi observado entre os meses de novembro a abril. Nos períodos de outono e inverno foram observados os menores números de moscas.

\section{Conclusões}

Com base nos resultados obtidos no presente estudo, chegou-se às seguintes conclusões:

a) nas condições do experimento, o núcleo homeopático apresentou efeito preventivo sobre o parasitismo por larvas de $D$. hominis;

b) nas condições do experimento, o núcleo homeopático não apresentou efeito preventivo sobre o parasitismo de $H$. irritans;

c) nos meses de outubro e novembro ocorreram os maiores índices de infestação por D. hominis;

b) entre os meses de novembro a abril ocorreram os maiores índices de infestação por H. irritans.

\section{Agradecimentos}

Ao Sr. Danilo Antônio Donin por permitir a condução do estudo na sua propriedade. Ao Instituto Tecnológico SIMEPAR pela assessoria prestada em relação aos dados climatológicos. 


\section{REFERÊNCIAS}

DITTRICH, R.L.; BARROS FILHO, I.R.; YORINOR, E.H.; SCHIMANSKI, N. Valores de referência para leucograma de bovinos da raça Holandesa Preta e Branca no Paraná. Revista do Setor de Ciências Agrárias, Curitiba, v. 15, n.2, p.207-211, 1996.

ESCOSTEGUY, A. A homeopatia em rebanhos leiteiros. A Hora Veterinária, Porto Alegre, n.131, p.51, 2003.

GOMES, A.; SOUSA, J.C.; RESENDE, A.M.; CURVO, J.B.E. Distribuição corporal e sazonalidade do berne (Larva de Dermatobia hominis) em bovinos tratados ou não com flor de enxofre. Pesquisa Agropecuária Brasileira, Brasília, v.23, n.8, p.825-829, 1988.

GOMES, A.; HONER, M.R.; SILVA, R.L. Intensidade Parasitária de larvas de Dermatobia hominis (L. Jr., 1781) (Diptera: Cuterebridae) em bovinos de diferentes raças criadas extensivamente na região de cerrado em Mato Grosso do Sul. Revista Brasileira de Parasitologia Veterinária, São Paulo, v.5, n.2, p.103-106, 1996.

GOMES, P.R. Espécies de Fanniidae (Diptera) vetoras de ovos de Dermatobia hominis (L. Jr., 1781) (Díptera: Cuterebridae), na região de Campo Grande, Mato Grosso do Sul. Campo Grande, 2001. 34f. Tese (Mestrado Interinstitucional em Biologia Parasitária), Universidade Federal do Mato grosso do Sul.

HOFFMANN, R.P. Diagnóstico de parasitismo veterinário. Porto Alegre: Sulina, 1987. 156p.

JAIN, N.C. Essentials of veterinary hematology. Philadelphia; Lea \& Febiger, 1993. 417p.

Recebido para publicação: 01/08/2004

Aprovado:
MARCONDES, C.B. Entomologia médica e veterinária. São Paulo: Editora Atheneu, 2001. 432p. OLIVEIRA, G.P. Dinâmica parasitária de bernes em bovinos. I. Incidência em relação ao decúbito. Pesquisa Agropecuária Brasileira. Série Zootecnia, Brasília, v.26, n.4, p.467-471, 1991.

PINTO, S.B.; SOCCOL, V.T.; VENDRUSCOLO, E.; ROCHADELLI, R.; RIBEIRO, P.B.; FREITAG, A.; HENEMANN,C.; UEMURA, M. Bioecologia de Dermatobia hominis (Linnaeus Jr., 1781) em Palotina, Paraná, Brasil. Ciência Rural, Santa Maria, v.32, n.5, p.821-827, 2002a.

PINTO, S.B.; SOCCOL, V.T.; ROCHADELLI, R.; ANDRADE, R.R.; MONTANUCCI, C.R. Marcadores parasitológicos e hematológicos para a seleção de bovinos resistentes a dermatobiose. Archives of Veterinary Science, Curitiba, v.7, n.1, p.21-25, 2002b.

REY, L. Parasitologia. 3.ed. Rio de Janeiro: Guanabara Koogan, 2001, 856p.

SUTHERST, R.W.; TOZER, R.S. Control of Buffalo fly (Haematobia irritans exigua de Meijere) on dairy and beef cattle using traps. Australian Journal Agriculture Research, Collingwood, n.46, p. 269284, 1995.

TOZER, R.S.; SUTHERS, R.W. Control of horn fly (Diptera: Muscidae) in Florida with Australian trap. Journal of Economic Entomology, Lanham, $n$. 89, p.415-420, 1996.

VIEIRA, S. Introdução a bioestatística. 2.ed. Rio de Janeiro: Ed. Campus, 1983. 